\title{
Teratoma cervical congênito gigante: relato de caso e revisão quanto às opções terapêuticas
}

\author{
Giant congenital cervical teratoma: case report and review about therapeutic options
}

Camila Ferro Miele ${ }^{1}$, Cristiane Metolina ${ }^{2}$, Ruth Guinsburg ${ }^{3}$

\section{RESUMO}

Objetivo: Relatar um caso de teratoma cervical congênito, destacando a gravidade e as dificuldades terapêuticas associadas.

Descrição do caso: Mãe de 30 anos, com gestação por fertilização assistida. Com 23 semanas, diagnosticada malformação cervical fetal à direita. Parto cesáreo por indicação fetal com 31 semanas. Recém-nascido masculino, peso ao nascer de $1800 \mathrm{~g}$, Apgar 4 e 9, com volumoso processo expansivo à direita, ocupando toda a região cervical, comprometendo a mandíbula e estendendo-se para o terço superior do tórax. Com 40 horas de vida, apresentou insuficiência cardíaca congestiva de alto débito por roubo de fluxo pelo tumor. A partir de 54 horas de vida, houve progressiva deterioração hemodinâmica e respiratória, com hipotensão, anúria e labilidade de oxigenação, refratárias às aminas vasoativas, reposição de volume e aumento do suporte ventilatório. Indicada abordagem cirúrgica para ressecção tumoral, todavia o paciente não apresentou estabilidade clínica que permitisse seu transporte ao centro cirúrgico e faleceu com 70 horas de vida.

Comentários: $\mathrm{O}$ caso demonstra as dificuldades relacionadas à abordagem pós-natal dos teratomas cervicais volumosos. Apesar do diagnóstico pré-natal, o paciente evoluiu com obstrução de vias aéreas, complicada por um choque cardiogênico refratário, que culminou no óbito. A abordagem intraparto é fundamental nesses pacientes, consistindo em exérese tumoral, enquanto a manutenção da circulação materno-fetal permite a oxigenação fetal contínua. A evolução neonatal no caso descrito é condizente com a literatura que mostra prognóstico reservado quando não é realizada a abordagem cirúrgica intraparto.

Palavras-chave: recém-nascido; anomalias congênitas; teratoma.

\section{ABSTRACT}

Objective: To report a case of congenital cervical teratoma, highlighting the severity and the therapeutic difficulties associated.

Case description: A 30-year old mother, with pregnancy by assisted fertilization. At 23 weeks, a cervical fetal malformation was diagnosed. A cesarean section was indicated with 31 weeks due to fetal distress. A male newborn infant with birth weight of $1800 \mathrm{~g}$ and Apgar score of 4/9 presented a large right cervical tumor, with extensions to the mandible and to the upper chest. Patient presented congestive heart failure due to flow steal by the tumor 40 hours after birth, which caused progressive respiratory, hemodynamic and renal deterioration refractory to vasopressors, volume replacement and increased ventilatory support. Tumor resection was indicated, but the clinical instability of the patient did not allow his transport to the operating room and the infant died 70 hours after birth.
Instituição: Departamento de Pediatria da Escola Paulista de Medicina da Universidade Federal de São Paulo (Unifesp), São Paulo, SP, Brasil

${ }^{1}$ Residente de Pediatria da Escola Paulista de Medicina da Unifesp, São Paulo, SP, Brasil

${ }^{2}$ Médica da Disciplina de Pediatria Neonatal da Escola Paulista de Medicina da Unifesp, São Paulo, SP, Brasil

${ }^{3}$ Professora Titular do Departamento de Pediatria da Escola Paulista de Medicina da Unifesp, São Paulo, SP, Brasil
Endereço para correspondência:

Camila Ferro Miele

Rua Rodrigo Cláudio, 308, apto. 41 - Aclimação

CEP 01532-020 - São Paulo/SP

E-mail: camilaferromiele@gmail.com

Conflitos de Interesse: nada a declarar

Recebido em: 17/11/2010

Aprovado em: 25/4/2011 
Comments: The case is representative of the difficulties related to postnatal treatment of bulky cervical teratomas. Despite prenatal diagnosis, the patient developed airway obstruction, complicated by refractory cardiogenic shock. The surgical approach during delivery is crucial for survival. Nowadays, management includes surgical removal of the tumor while maintaining the maternal-fetal circulation, allowing continuous fetal oxygenation. The clinical course described in the case is consistent with the literature that reports poor prognosis when the intra-partum surgical approach is not performed.

Key-words: infant, newborn; congenital abnormalities; teratoma.

\section{Introdução}

Teratomas são tumores originados a partir de células totipotentes, apresentando componentes celulares remanescentes de mais de uma camada germinativa ${ }^{(1-3)}$. São mais comuns na primeira e segunda infâncias e localizam-se, com maior frequência, nas gônadas e na região sacrococcígea, respectivamente em adultos e crianças. A localização cervical é incomum e, quando ocorre, a região cervicotireoidiana é a mais acometida ${ }^{(3,4)}$.

Os teratomas cervicofaciais em neonatos compreendem 1,5 a 5,5\% dos teratomas e apresentam incidência de 1:20.000 a 1:80.000 nascidos $\operatorname{vivos}^{(1,2,5)}$. Esses tumores geralmente apresentam-se de forma isolada, sem associação com anomalias cromossômicas ou outras malformações congênitas ${ }^{(5)}$. Macroscopicamente, os teratomas cervicais são massas bem circunscritas, volumosas, com componente sólido e cístico, variando de 5 a $12 \mathrm{~cm}$ em seus maiores diâmetros ${ }^{(1,5,6)}$. Apesar de serem neoplasias benignas em $95 \%$ dos casos, os teratomas cervicais não tratados levam ao óbito em até $80 \%$ dos casos, devido principalmente à insuficiência respiratória obstrutiva ${ }^{(1,3,5,7)}$.

A classificação anatomopatológica dos teratomas mais aceita é a de Kooijman, de 1988, que os divide em teratomas maduros, com tecidos tumorais bem diferenciados; teratomas imaturos, com presença de tecido neuroectodérmico imaturo; e teratomas combinados, nos quais há associação com tumores de células germinativas ${ }^{(4)}$. Os teratomas imaturos são analisados, ainda, conforme a extensão do componente primitivo $^{(4)}$. Não há evidências de que o grau de imaturidade interfira no prognóstico dos teratomas cervicofaciais tratados, nem mesmo que possa indicar um potencial comportamento maligno ${ }^{(3,4,8)}$.
Dependendo da localização e do tamanho tumoral, a ultrassonografia (USG) pré-natal pode ser diagnóstica e mostrar ainda a presença de polidrâmnio em um terço dos casos. O exame passa a ser mais sensível a partir de 15 semanas de idade gestacional ${ }^{(1,3,5,6,9)}$. A existência do polidrâmnio pode ser explicada pela hiperperfusão da placenta devido ao aumento do volume sanguíneo ocasionado pelo tumor ou devido à dificuldade do feto em deglutir o líquido amniótico por compressão tumoral, sendo a última hipótese a mais aceita ${ }^{(4)}$. Seu diagnóstico diferencial deve ser realizado com outras massas cervicais, como higroma cístico, bócio fetal, cisto dermoide, cisto tireoglosso, cisto congênito de tireoide, torcicolo congênito, linfangioma, cisto branquial, neuroblastoma, carcinoma de tireoide, bócio congênito e tumor de parótida ${ }^{(4,7,9)}$.

A complicação mais temida dos teratomas cervicais é a insuficiência respiratória causada pela compressão traqueal pelo tumor. A obstrução das vias aéreas impõe, muitas vezes, a intervenção imediata, ainda na sala de parto, com intubação orotraqueal ou traqueostomia. Caso o neonato não demonstre sinais de insuficiência respiratória logo ao nascimento, a intubação orotraqueal deve ser realizada de forma eletiva, o mais precocemente possível ${ }^{(1,4,6,7,10)}$. A via de parto preferencial é a cesariana.

A ressonância nuclear magnética pode propiciar, nos casos em que a programação cirúrgica é eletiva, um melhor planejamento operatório, auxiliando na verificação dos planos de dissecção e separação das estruturas vitais ${ }^{(4,6,7,10)}$. O tratamento por meio de ressecção cirúrgica precoce de todo o tumor constitui-se na abordagem mais adequada dessa patologia, pois a degeneração maligna ocorre em até $90 \%$ dos casos não tratados até a adolescência ou vida adulta. A excisão cirúrgica geralmente é curativa ${ }^{(1,4-7)}$. Esquemas de quimioterapia ou radioterapia são reservados apenas para os casos dos teratomas cervicais malignos, de doença disseminada ou de tumor invasivo residual, porém não há quimioterápicos pré-estabelecidos para esses $\operatorname{casos}^{(7,9)}$.

O prognóstico é determinado pelo grau de desconforto respiratório ao nascimento, que, em $50 \%$ dos casos, é intenso devido à compressão da via aérea pelo tumor, caracterizando a complicação pós-natal mais frequente e grave. Outros critérios prognósticos incluem a presença de anomalias associadas, desenvolvimento de hidropisia fetal e idade da apresentação tumoral ${ }^{(4,6,11)}$. Dentre as opções de intervenção imediata e ressecção tumoral não eletiva, destacam-se o tratamento extraútero intraparto (EXIT Procedure) e a operação com suporte placentário (OOPS 
Procedure). O EXIT é realizado após a exteriorização parcial do feto da cavidade uterina, mantendo intacta a circulação uteroplacentária ${ }^{(5,7,9)}$. A mortalidade neonatal dos teratomas cervicais sem a realização do EXIT cursa em torno de 80 a $100 \%$, diminuindo para 9 a $17 \%$ com o EXIT e tratamento definitivo, dependendo do envolvimento de estruturas nobres ${ }^{(1,5,9)}$.

O presente estudo visou relatar um caso de teratoma cervical congênito, destacando a gravidade e as dificuldades terapêuticas associadas.

\section{Descrição do caso}

Mãe de 30 anos, portadora de endometriose e síndrome dos ovários policísticos, primigesta, com gestação por fertilização assistida. Com idade gestacional de 23 semanas e dois dias, foi diagnosticada malformação cervical fetal à direita, com dimensões de 5,3x5,0x4,1cm. Realizada a ressonância nuclear magnética fetal com 31 semanas e dois dias, que mostrou massa em íntimo contato com a base do crânio na fossa média (fossa temporal e mastoide direitas), côndilo, ramo e ângulo mandibulares direitos, com sinais de erosão óssea dos mesmos. Ausência de planos de clivagem com assoalho bucal, podendo envolver a língua e glândula tireoide, não visualizados no estudo. Acentuado deslocamento da laringe e traqueia para a esquerda, com sinais de estreitamento na transição laringotraqueal. Caudalmente, havia comprometimento do mediastino superior desde o terço superior do tórax.

Com 31 semanas e quatro dias, a gestante entrou em trabalho de parto prematuro sem causa aparente. O concepto nasceu de parto cesáreo por indicação fetal.

Tratava-se de um recém-nascido pré-termo, do sexo masculino, com peso de nascimento de $1800 \mathrm{~g}$, Apgar no $1^{\circ}$ e $5^{\circ}$ minuto de 4 e 9 , respectivamente. O paciente apresentava volumoso processo expansivo à direita, ocupando toda a extensão da região cervical, com crescimento exofítico e comprometimento mandibular, estendendo-se para o terço superior do tórax. Macroscopicamente, a tumoração mostrava-se lobulada, ricamente vascularizada, com áreas esparsas de necrose e saída de secreção serossanguinolenta, sem outras malformações associadas (Figura 1).

O paciente evoluiu com desconforto respiratório obstrutivo logo ao nascimento, optando-se por intubação orotraqueal em sala de parto. Durante o procedimento, houve grande dificuldade de retificação de vias aéreas, restrita visualização da traqueia pela compressão tumoral e impossibilidade de posicionamento adequado do recém-nascido devido à extensa massa cervical. Após três tentativas, a intubação foi realizada com cânula orotraqueal número 2,5.

Durante as primeiras 40 horas de vida, o neonato permaneceu hemodinamicamente estável, em suporte ventilatório mínimo, intubado por obstrução de vias aéreas, sem doença pulmonar de base. Com 40 horas de vida, iniciou comprometimento cardiovascular com sinais de baixo débito sistêmico. Foi feita a hipótese de insuficiência cardíaca congestiva de alto débito, por roubo de fluxo pelo tumor, sendo iniciado suporte inotrópico com dobutamina e, após persistência da instabilidade dos parâmetros hemodinâmicos, indicada a associação com a dopamina. Simultaneamente, foram aumentados parâmetros respiratórios. A partir de 54 horas de vida, mostrou progressiva deterioração hemodinâmica e respiratória, com hipotensão, anúria e labilidade de oxigenação, refratárias às aminas vasoativas, à reposição de volume e ao aumento do suporte ventilatório. Com 63 horas de vida, o paciente apresentava-se em mau estado geral, com vasculite e escleredema generalizados, hipoxemia e choque refratário, sendo introduzidos milrinona e noradrenalina, e realizado $100 \mathrm{mg} / \mathrm{kg}$ de surfactante pulmonar. Foi indicada abordagem cirúrgica por equipe neonatal e cirúrgica, para ressecção tumoral e consequente estabilização hemodinâmica do paciente; todavia, o paciente não apresentou estabilidade clínica mínima que permitisse seu transporte ao centro cirúrgico, falecendo com 70 horas de vida.

O paciente foi submetido à necrópsia, que evidenciou massa cervical mediana, medindo $15 \times 10 \times 7 \mathrm{~cm}$, ulcerada, sólidocística, com deslocamento traqueal e intensa vascularização,

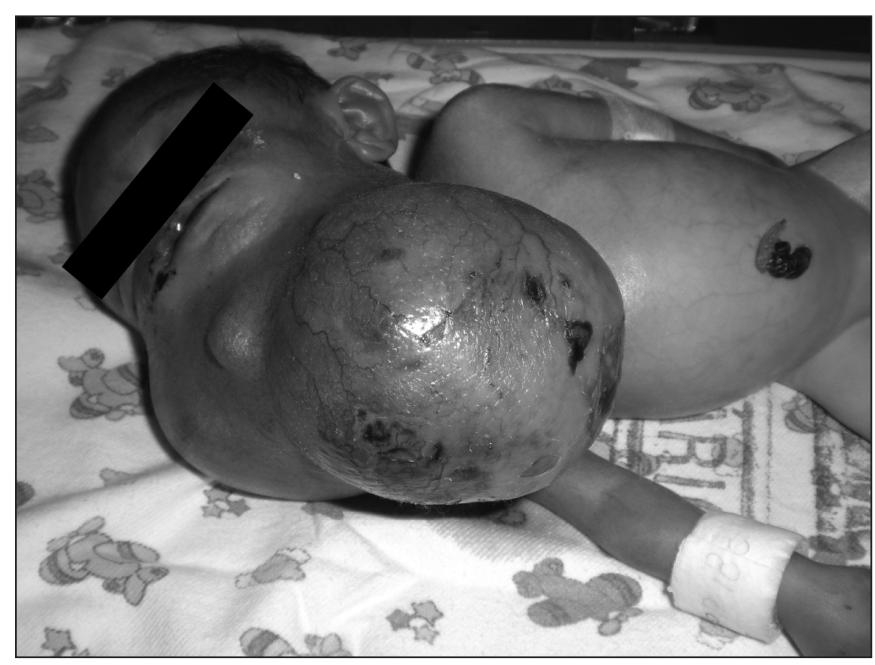

Figura 1 - Recém-nascido com massa tumoral cervical gigante, com regiões de sangramento e necrose (com permissão dos pais) 
além de sinais de dilatação de todo o território venoso supradiafragmático por congestão. O estudo anatomopatológico revelou como causa imediata do óbito a insuficiência cardíaca de alto débito, com roubo vascular pela tumoração, associada à compressão de grandes vasos cervicais. À microscopia, havia agrupamentos celulares de neuroepitélio, tecido glandular e cartilagíneo, compatível histologicamente com teratoma cervical congênito grau III de imaturidade.

\section{Discussão}

O caso aqui relatado demonstra as dificuldades relacionadas à abordagem pós-natal dos teratomas cervicais. O paciente em questão, apesar do diagnóstico pré-natal, evoluiu com obstrução de vias aéreas de difícil abordagem clínica, complicada por um choque cardiogênico refratário, que culminou no óbito.

Os teratomas cervicais podem desencadear diversos sintomas referentes à compressão de estruturas adjacentes ao tumor, como a obstrução esofágica, com distúrbios de deglutição, e hipoplasia de laringe, mandíbula e de cordas vocais por compressão da via aérea. Já a hipoplasia pulmonar pode ocorrer de modo secundário à compressão do pulmão pelo tumor $^{(5,10-12)}$. Além desses, a presença de hidropisia fetal é relatada por Hirose et al. em conceptos portadores de teratoma cervical $^{(13)}$. Porém, a consequência mais temida ao nascimento é a obstrução das vias aéreas, com sintomas respiratórios graves e potencialmente fatais, devido à compressão da traqueia e consequente dano hipóxico-isquêmico ao concepto ${ }^{(1,7,9,12)}$. Ainda, como complicações associadas ao momento do parto, citam-se a distócia de apresentação e funcional, secundárias à hiperextensão do pescoço e/ou ao volume tumoral, sendo indicado geralmente o parto cesáreo ${ }^{(6,9,10)}$. Finalmente, devido ao rápido crescimento tumoral, pode ocorrer consumo de fatores coagulação, com trombocitopenia e coagulopatia na vida fetal e pós-natal ${ }^{(4)}$.

No caso aqui apresentado, chama atenção o grande volume do tumor, com consequente insuficiência respiratória aguda logo ao nascimento, tendo necessitado de intubação orotraqueal com ventilação mecânica invasiva. O desenvolvimento de técnicas mais apuradas de diagnóstico pré-natal, destacando-se as técnicas de diagnóstico por imagem e o aperfeiçoamento das técnicas cirúrgicas para garantir a permeabilidade das vias aéreas pérvia após o nascimento, garante a diminuição da morbidade e a mortalidade dos teratomas nas últimas décadas. O diagnóstico de via aérea obstruída é geralmente feito na $30^{\mathrm{a}}$ semana de idade gestacional, com auxílio da ultrassonografia e da ressonância magnética ${ }^{(7,9)}$.

A abordagem intraparto é fundamental em pacientes portadores de teratomas cervicais volumosos, em que a obstrução das vias aéreas é importante. Uma das principais formas de intervenção ao nascimento se dá por meio da manutenção da circulação materno-fetal, a qual permite uma oxigenação fetal contínua, enquanto se garante a permeabilidade da via aérea. O cordão umbilical é clampeado apenas após a garantia de que a via aérea está pérvia, por meio da intubação traqueal ou da traqueostomia ${ }^{(5,9)}$. Entre as opções de intervenção imediata, não eletiva, destacam-se o tratamento extraútero intraparto (EXIT procedure, EXIT do inglês ex utero intrapartum treatment) e a operação com suporte placentário (OOPS procedure) $)^{(5,7,9)}$. Quando a abordagem ao nascimento é a extração completa do tumor por meio de OOPS procedure, é feita a retirada completa do feto e do cordão umbilical do útero, mantendo-se, também, a circulação materno-fetal ${ }^{(9,11)}$. No entanto, o procedimento que tem sido mais estudado é o EXIT.

Para a realização do EXIT procedure, o parto deve ser cesáreo, com retirada da cabeça, pescoço e braço fetal, expondo-os no campo operatório. Nesse momento, é indicada a intubação traqueal ou a traqueostomia, garantindo a permeabilidade da via aérea, enquanto a circulação materno-fetal é mantida. Durante o EXIT, a depressão respiratória do recém-nascido consequente à anestesia materna não constitui problema, pois o procedimento se conclui com a intubação e a ventilação do paciente. Outro aspecto importante no EXIT relaciona-se à manutenção do volume uterino em nível apropriado para preservar a perfusão placentária. O tempo de duração do procedimento é de aproximadamente 1 hora, desde que não haja necessidade de ressecção de um grande teratoma cervical no período intraparto ${ }^{(4-6,9)}$.

Em ambos os procedimentos, OOPS e EXIT, as complicações pós-operatórias ao recém-nascido incluem danos aos nervos laringeo recorrente, hipoglosso e mandibular marginal $^{(4,9,11)}$.

O tratamento definitivo de escolha é a ressecção cirúrgica do tumor com manutenção do aporte respiratório e da estabilidade hemodinâmica do neonato, associada à quimioterapia ou à radioterapia nos casos de lesões não ressecáveis e teratomas malignos ${ }^{(1,4,5,7)}$. Quando a exérese do tumor é eletiva, a avaliação pré-operatória pode incluir uma radiografia de tórax e uma tomografia computadorizada de cabeça, pescoço e tórax, para fornecer informações quanto à invasão e tumoral e à extensão do tumor ${ }^{(6,9)}$. 
O prognóstico depende do grau de compressão da via aérea ao nascimento, da presença de outras anomalias, do desenvolvimento de hidropsia e também da idade de apresentação ${ }^{(1,11)}$. Se a via aérea é garantida e o tumor removido no período neonatal imediato, o resultado é excelente, com uma taxa de sobrevivência superior a $85 \%{ }^{(11)}$.

Pode-se concluir que os teratomas cervicais congênitos requerem o diagnóstico pré-natal, o que propicia a abordagem

\section{Referências bibliográficas}

1. Schmidt M, Wolke S, Hübler A, Eckoldt F, Schultze-Mosgau S. Successful treatment of a huge congenital cervical teratoma. J Craniofac Surg 2009;20:1277-80.

2. Makary R, Wolfson D, Dasilva V, Mohammadi A, Shuja S. Intramedullary mature teratoma of the cervical spinal cord at C1-2 associated with occult spinal dysraphism in an adult. Case report and review of the literature. J Neurosurg Spine 2007;6:579-84.

3. Bianchi B, Ferri A, Silini EM, Magnani C, Sesenna E. Congenital cervical teratoma: a case report. J Oral Maxillofac Surg 2010;68:667-70.

4. Vandenhaute B, Leteurtre E, Lecomte-Houcke M, Pellerin P, Nuyts JP, Cuisset $\mathrm{JM}$ et al. Epignathus teratoma: report of three cases with a review of the literature. Cleft Palate Craniofac J 2000;37:83-91.

5. Bleggi-Torres LF, Dellê LA, Urban C de A, Araki LT. Cervicofacial teratoma in neonates. J Pediatr (Rio J) 1998;74:149-52.

6. Johnson N, Medd L, Shah PS, Shannon P, Campisi P, Windrim R et al. A challenging delivery by EXIT procedure of a fetus with a giant cervical teratoma. J Obstet Gynaecol Can 2009;31:267-71.

7. Nascimento GC, Souza AS, Lima MM, Guerra GV, Meneses JA, Cardoso AS et al. Estratégia de conduta intraparto no teratoma cervical congênito: procedimento EXIT (Tratamento Extra-Útero Intraparto). Acta Med Port 2007;20:221-7. apropriada ao nascimento. É fundamental o planejamento préoperatório com equipe multidisciplinar, visando à estabilização imediata do neonato na sala de parto. Faz-se necessário, também, assegurar à mãe quanto ao risco quase ausente de futuras gestações com a mesma doença ${ }^{(14)}$. A evolução neonatal, no caso aqui descrito, é condizente com os dados encontrados na literatura que mostram prognóstico reservado diante de teratomas gigantes sem a abordagem cirúrgica na sala de parto.

8. Elmasalme F, Giacomantonio M, Clarke KD, Othman E, Matbouli S. Congenital cervical teratoma in neonates. Case report and review. Eur J Pediatr Surg 2000;10:252-7.

9. Mann JR. Germ cell tumours of childhood. In: Peckhann M, Pinedo HM, Veronesi U, editors. Oxford textbook of Oncology. New York: Oxford University Press; 1995. p. 2022-36.

10. Braga Ade F, Frias JA, Braga FS, Rousselet MS, Barini R, Sbragia L et al. Anesthesia for ex utero intrapartum treatment of fetus with prenatal diagnosis of cervical hygroma: case report. Rev Bras Anestesiol 2006; 56:278-86.

11. Afolabi IR. Sacrococcygeal teratoma: a case report and a review of literature. Pac Health Dialog 2003;10:57-61.

12. Shah SI, Holterman AX, Licameli GR. Congenital cervical teratoma: airway management and complications. Otolaryngol Head Neck Surg 2001;124:53-5.

13. Hirose S, Sydorak RM, Tsao K, Cauldewell CB, Newman KD, Mychaliska GB et al. Spectrum of intrapartum management strategies for giant fetal cervical teratoma. J Pediatr Surg 2003;38:446-50.

14. Muscatello L, Giudice M, Feltri M. Malignant cervical teratoma: report of a case in a newborn. Eur Arch Otorhinolaryngol 2005;262:899-904. 